\section{Innovation methods}

\section{CAN SYNTHETIC CONTROLS IMPROVE CAUSAL INFERENCE IN INTERRUPTED TIME SERIES EVALUATIONS OF PUBLIC HEALTH INTERVENTIONS?}

${ }^{1}$ Michelle Degli Esposti, ${ }^{1}$ Thees Spreckelsen, ${ }^{2}$ Antonio Gasparrini, ${ }^{3}$ Douglas I Wiebe, ${ }^{4}$ Alexa $\mathrm{R}$ Yakubovich, 'David K Humphreys. 'Department of Social Policy and Intervention, University of Oxford; ${ }^{2}$ Department of Public Health, Environments and Society, London; ${ }^{3}$ Department of Biostatistics and Epidemiology, University of Pennsylvania; ${ }^{4}$ Centre for Urban Health Solutions, Li Ka Shing Knowledge Institute, St Michael's Hospital, Toronto, Canada

\subsection{6/injuryprev-2020-savir.37}

Statement of Purpose Interrupted time series (ITS) designs are a valuable quasi-experimental approach for evaluating public health interventions. ITS extends a single group pre-post comparison by using multiple timepoints to control for underlying trends. But history bias - confounding by unexpected events occurring at the same time of the intervention - threatens the validity of this design and limits causal inference. Synthetic control methodology (SCM), a popular data-driven technique for deriving a control series from a pool of unexposed populations, is increasingly recommended. We aimed to evaluate if and when SCM can strengthen an ITS design.

Methods/Approach First, we summarise the main observational study designs used in evaluative research, highlighting their respective uses, strengths, biases, and design extensions. Second, we outline when the use of SCM can strengthen ITS studies and when their combined use may be problematic. Third, we provide recommendations for using SCM in ITS and, using a real-world example of an evaluation of Florida's Stand Your Ground laws on homicides, we illustrate the potential pitfalls of using a data-driven approach to identify a suitable control series.

Results Our real-world evaluation demonstrates that the benefits of SCM in ITS depends on the nature of the time-varying confounding which presents the most plausible threat to the study's validity. We emphasise the importance of theoretical approaches for informing study design and argue that synthetic control methods are not always well-suited for minimising critical threats to ITS studies.

Conclusions Advances in SCM bring new opportunities to conduct rigorous research in evaluating public health interventions. However, incorporating synthetic controls in ITS studies may not always nullify important threats to validity nor improve causal inference.

Significance and Contributions to Injury and Violence Prevention Science We provide important methodological recommendations to guide advancement in the science of injury and violence prevention.

\section{Motor vehicle crashes: epidemiology and interventions}

\section{IMPACT OF MOTOR VEHICLE CRASHES ON LONG-TERM HEALTHCARE UTILIZATION}

Larry Cook, Jesse Norris, Emily Startup, Cody Olsenc. University of Utah Department of Pediatrics

10.1136/injuryprev-2020-savir.38
Statement of Purpose The impact of a motor vehicle crash (MVC) on health can have effects beyond injuries treated immediately following the crash. Understanding the long-term effects of an MVC can help develop programs to assist injured persons while having minimal impact on the healthcare system.

Methods We probabilistically linked MVC records from 2011 and 2015 to emergency department (ED) and hospital discharge (inpatient) records from 2010 to 2016. To assess the long-term impact of an MVC, ED and inpatient visits up to a year following the data of the crash were considered matches. ED and inpatient visits in the year preceding the crash were also linked to use as a comparison of healthcare utilization before the event. Several confounders were assessed for their impact on post-crash healthcare usage, including age, sex, injury severity, and comorbidities identified on the hospital record at the time of the crash.

Results There were 652,486 persons involved in MVCs, of these $68,546(10.5 \%)$ persons linked to an ED or inpatient record associated with their MVC and comprised our study population. A higher percentage of persons had hospital treatment in the year following their crash compared to the year before [19,568 (28.5\%) vs. 16,500 (24.1\%), McNemar's pvalue $<0.001)$. Increased hospitalization usage was consistent across all ages, sexes, and comorbidities. However, post-MVC healthcare resulted in higher median charges $(\mathrm{p}<0.001)$ and lengths of stay (LOS) $(\mathrm{p}<0.001)$. This relationship increased with age where those 65-89 years had pre-MVC median charges of $\$ 3,497$ and LOS of 2 but post-MVC median charges of $\$ 4,330$ and LOS of $5(\mathrm{p}<0.001)$. Similarly, those with comorbidities experienced higher post-MVC charges $(\$ 3,380$ pre vs. $\$ 4,362$ post, $\mathrm{p}<0.001)$.

Significance of Contributions MVCs have an impact beyond the event itself. Those who are most fragile, such as older persons and those with comorbidities, may be differentially impacted.

\section{Mental health consequences of violence}

\section{THE CONTRIBUTION OF NEIGHBORHOOD CHARACTERISTICS TO PSYCHOLOGICAL SYMPTOM SEVERITY IN A COHORT OF INJURED BLACK PATIENTS}

Marta Bruce, Connie Ulrich, Justine Shults, Douglas J Wiebe, Therese S Richmond. University of Pennsylvania, Schools of Medicine and Nursing

\subsection{6/injuryprev-2020-savir.39}

Background Traumatic injury is not evenly distributed across race and class in America. Black men are marginalized in society, often live in disadvantaged neighborhoods, and are at higher risk for injury mortality and ongoing physical and psychological problems following injury, including post-traumatic stress disorder (PTSD) and depression. While much research has examined individual factors associated with increased post-injury psychological symptoms, the contribution of the social and physical environment has been relatively-understudied.

Purpose The purpose was to examine the contribution of neighborhood characteristics to PTSD and depressive symptom severity in Black men following serious injury.

Methods A secondary analysis of 451 seriously injured men living in Philadelphia were drawn from a prospective cohort 
study. Study participants were linked by a geographic information system (GIS) to characteristics in their neighborhood in order to spatially model the factors that contribute to increased psychological symptom severity at 3 months postinjury. Factor analysis identified underlying constructs of 32 neighborhood predictors, which were entered in a generalized estimating equation (GEE) regression analysis. Covariates included mechanism of injury, severity of injury, age, and health insurance.

Results Neighborhood characteristics loaded onto 4 constructs: neighborhood disconnectedness; concentrated disadvantage; crime, violence, and vacancy; and race/ethnicity. Higher PTSD symptom severity was reported by $36.8 \%$ and associated with neighborhood crime, violence, and vacancy, and higher depressive symptom severity was reported by $30.4 \%$ and associated with neighborhood disconnectedness. Both PTSD and depressive symptoms were associated with intentional mechanisms of injury, such as gunshot wounds, stabbing, and assault; and with having Medicaid or no insurance. Higher severity of injury was associated with depressive symptoms.

Conclusions Findings suggest that neighborhood characteristics such as neighborhood disconnectedness and crime, violence, and vacancy have measurable associations with psychological symptoms after injury. The evidence supports the need for interventions to improve post-injury mental health by modifying the urban environment.

\section{Equity and methods}

\section{IMPLICATIONS FOR HEALTH EQUITY AND LESSONS LEARNED FROM A COMMUNITY-ENGAGED MODIFIED DELPHI PROCESS AND NOMINAL GROUP TECHNIQUE}

\author{
1,2Kelsey Conrick, ${ }^{2}$ Brianna Mills, ${ }^{1,2}$ Frederick Rivara, ${ }^{1,2}$ Monica Vavilala. ${ }^{1}$ University of \\ Washington School of Social Work; ${ }^{2}$ University of Washington Harborview Injury Prevention \\ and Research Center
}

10.1136/injuryprev-2020-savir.40

Statement of Purpose Achieving injury-related health equity requires community-informed research and close collaboration among diverse (in lived experience, education, geography, etc.) stakeholders. Differing priorities, knowledge, and communication styles can inhibit participation in the consensus-building process, inhibiting the progress of research and development of successful interventions. Standardized, evidence-based procedures are needed to achieve consensus among diverse groups of stakeholders.

Methods/Approach We use a novel, rigorous process involving a combined Delphi process and Nominal Group Technique (NGT) to describe the development of recommendations for the pre-hospital care of the spine-injured athlete as a framework for achieving consensus among a group of stakeholders and community members. We outline our process and discuss lessons learned and implications for health equity.

Results We used an online modified Delphi process to achieve consensus on the research questions of priority to stakeholders. After conducting an independent systematic review, we presented our findings at an in-person meeting for twenty stakeholders. We used NGT to develop and agree upon protocol recommendations. Throughout this process, we learned several lessons. Most crucial to the success of the in-person NGT process is a reputable, experienced facilitator capable of managing complex group dynamics and adhering to agreed-upon rules of engagement. Clarification of research jargon and transparency throughout the research process engages stakeholders of all levels of experiences. Finally, innovative uses of technology may expedite the otherwise cumbersome process of providing opinions and voting to achieve consensus.

Conclusions While many researchers appreciate the value of community and stakeholder input towards the achievement of injury-related health equity, concern about managing and facilitating this process may limit its efficiency, inhibiting stakeholder investment in the research process.

Significance and Contributions to Injury and Violence Prevention Science Developing and implementing a well-informed, evidence-based protocol for achieving consensus may facilitate community-engaged research aimed to achieve injury-related health equity.

\section{Sports injury}

\section{CONCUSSION PREVENTION AWARENESS IN MIDDLE SCHOOL SPORTS}

Paula Gildner. University of North Carolina Injury Prevention Research Center

10.1136/injuryprev-2020-savir.41

Statement of Purpose Limited research specific to middle school (MS) sports on concussion prevention exists. We examined general perceptions, knowledge, attitudes, and communication constructs related to concussion prevention among MS sports stakeholders.

Methods/Approach Semi-structured interviews with coaches/ school staff $(n=6)$, parents, $(n=10)$ and athletes $(n=10)$ were conducted as part of a larger study throughout the 20182019 academic year at four North Carolina MSs. Interviews were transcribed verbatim; data were analyzed using the consensual qualitative tradition where a codebook was created using four progressive stages.

Results Three major themes were identified. First, there was a general lack of infrastructure and context surrounding sport safety and concussion prevention. Participants reported that the emphasis is on athletes simply exploring the varied sport options offered at the MS level, good sportsmanship and sport enjoyment; sport safety and concussion prevention was not a high concern. Second, there was limited awareness by stakeholders at the MS level concerning the importance of concussion prevention. Participants noted that concussion prevention is rarely discussed at the MS level and does not resonate with stakeholders. Third, there was ineffective communication between stakeholders regarding several topics, including concussion prevention. Participants noted that communication between the Athletic Directors, coaches, parents, and athletes was focused mainly on logistics, e.g., schedule changes. In addition, participants reported varied modes of communication utilized by school, of which most reportedly posed challenges for key stakeholders.

Conclusions These findings suggest a need to develop a cohesive strategy specific to the MS setting to disseminate concussion prevention information. Researchers and other MS sport stakeholders should collaborate to develop methods for sharing concussion prevention information at this level. 